\title{
Allergiker in die Ämter und Ministerien!
}

$\mathrm{D}$ ie Allergologie hat in Deutschland immer noch nicht die wissenschaftliche und wissenschafts- sowie hochschulpolitische Bedeutung erlangt, die ihr angesichts der Prävalenz der allergischen Erkrankungen von insgesamt rund $40 \%$ der Bevölkerung gebührt. Die Ursachen hierfür sind vielfältig - nicht zuletzt begründet in der organ- und fachübergreifenden Symptomatik, die nicht in das deutsche Facharztsystem passt. Dennoch ist die Politik längst auf diese Erkrankungen aufmerksam geworden - siehe das Interesse von Bundesminister Seehofer, bevor er nach München gerufen wurde.

Mit der aktuellen Situation, in der die wichtigsten Basismedikamente für allergische Erkrankungen, die Antihistaminika, von Ausnahmen abgesehen als Fall für eine rezept- und vergütungsfreie Selbstversorgung bewertet werden, können die Allergologen „Man kann sich nur wünschen, und deren Verbände nicht dass endlich von Allergien zufrieden sein. Man kann betroffene Entscheidungs- sich nur wünschen, dass tråger in die entsprechenden endlich von Allergien beGremien gewählt werden。“ troffene Entscheidungsträger in die entsprechenden Gremien gewählt werden. Da diese erst ab dem Geburtsjahrgängen 1960 und folgende weiter zunehmen, ist wahrscheinlich noch etwas Geduld geboten.

International haben deutsche Allergologen dagegen längst eine führende Rolle erlangt. Als Beispiele seien nur die neuesten Studien über die sublinguale Immuntherapie genannt, publiziert unter deutschsprachigen Erstautoren (Bufe, Bochum, und Wahn, Berlin) oder die kürzliche Würdigung der epidemiologischen Forschungen von Prof. Dr. Erika von Mutius, München, in Lancet.

Das Allergo Journal hat sich als das Organ für die wichtigen allergologischen Aktivitäten in Deutschland seit Jahren fest etabliert. Dies wird auch in diesem Heft deutlich: „Im Blickpunkt“ stehen Adjuvanzien zur spezifischen Immuntherapie, die nicht nur wichtige Bestandteile der verfügbaren Präparate sind, sondern auch weitere Fortschritte versprechen (S. 261).

Das europäische Posisitonspapier der EAACI zur Problematik der IgG-Antikörperbestimmung gegen Allergene, dem sich die deutschsprachigen

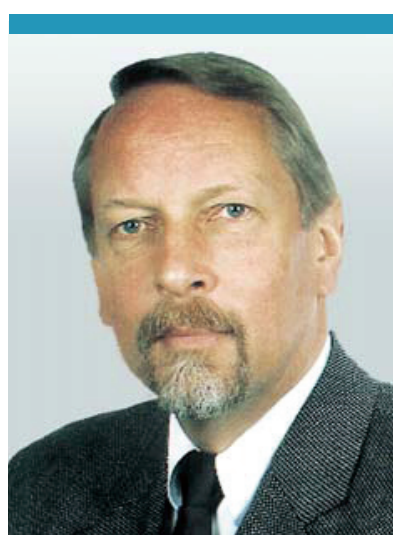

Prof. Dr. Gerhard SchultzeWerninghaus, Abteilung für Pneumologie, Allergologie und Schlafmedizin, BG-Kliniken Bergmannsheil - Universitätsklinik, Bochum

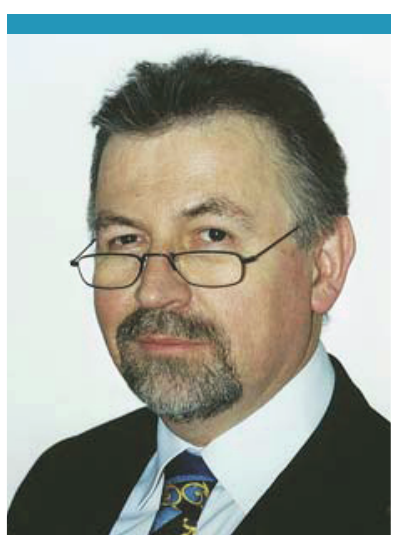

Prof. Dr. Bernhard Przybilla, Klinik und Poliklinik für Dermatologie und Allergologie, Klinikum der Ludwig-MaximiliansUniversität, München

allergologischen Fachgesellschaften anschließen, wird in diesem Heft publiziert (S. 267). IgG-Bestimmungen haben keinerlei krankheitsspezifische Bedeutung, werden aber dennoch häufig durchgeführt, zum Teil verbunden mit umfangreichen Diätempfehlungen, die nicht nur unbegründet sind, sondern sogar gesundheitsschädlich sein können. Alle informierten Ärzte sind aufgefordert, der Abzocke und Verunsicherung der Patienten durch IgG-Antikörpertests eine Absage zu erteilen.

Der Leser wird weiter in Form von Kurzbeiträgen informiert über die dritte Jahrestagung der Deutschen Gesellschaft für Epidemiologie von 24. bis 27. September 2008, auf der allergologische Themen eine wichtige Rolle spielten (ab S. 274).

Den Abschluss bildet der tauchmedizinische CME-Beitrag, der dazu beitragen soll, die verbreitete Unsicherheit bei der Beratung von allergischen Patienten, die sich in die Tiefe wagen wollen, abzubauen (S. 286).

Wir wünschen Ihnen eine spannende Lektüre!

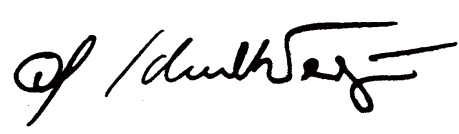

Prof. Dr. G. Schultze-Werninghaus

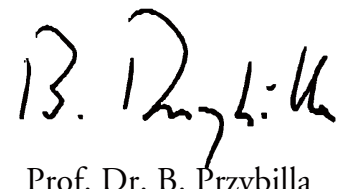

